L. Hermann: Ueber Brechung bei schiefer Incidenz, etc.

(Aus dem physiologischen Laboratorium in Zürich.)

\title{
Ueber Brechung bei schiefer Incidenz, mit besonderer Berücksichtigung des Auges.
}

\author{
III. Theil. (S e hlus s.) \\ (Vergl. dies Archiv Band XVIII, p. 443 und Bd. XX, p. $370^{1}$ ).) \\ Von
}

\section{Hermann.}

11. Zusätze zu den beiden ersten Theilen dieser Arbeit.

Unablässige Beschäftigung mit dem vorliegenden Gegenstande, welche seit Jahren meine Mussestunden ausfüllt, hat mich auf eine Anzahl von Vereinfachungen und interessanten neuen Beziehungen geführt, welche kurz mitgetheilt werden mögen, ehe die Aufgabe selber zum Abschluss gebracht wird. Die Beschränkung auf kleine Incidenzwinkel habe ich seit der letzten Publication principiell aufgegeben, so dass alles Folgende in aller Strenge, ohne irgend welche Vernachlässigung kleiner Grössen, gültig ist (mit Ausnahme von Gleichung 37).

a. Einführung einiger Bezeichnungen.

Die bisher mit dem Index 2 bezeichneten Brennlinien, resp. deren Lagen, sollen fortan im Texte als Hauptsehnitt-Brennlinien, und in den Ausdrücken durch den Index * bezeichnet werden, und die mit dem Index 1 figurirenden als Kreuzschnitt-

1) Im zweiten Theil ist durch einen Schreibfehler in den Gleichungen 78 (Band $\mathrm{XX}$, p. 383) rechterseits der Factor $\frac{1}{\mathrm{~A}}$ weggelassen. 
Brennlinien, in den Ausdrücken durch $\dagger$ gekennzeichnet. Die Benennungen rechtfertigen sich von selbst, und die Abschaffung der Indices 1 und 2 rechtfertigt sich dureh das Bd. XX, p. 376 Anm. Gesagte; blosse Vertauschung der Indices würde Verwirrung hervorrufen.

b. Verbesserte Form der Ausdrücke für eine einzelne Fläche.

Sind die Winkel zwischen Strahlenbündel und Einfallsloth $\varphi$ auf der convexen, $\alpha$ auf der concaven Seite, $e$ und $f$ die entsprechenden Bildweiten, ferner das Brechungsverhältniss $n$, so dass $\sin \varphi=n \sin \alpha$, endlich $r$ der Krümmungsradius, so hatten wir die Beziehungen gefunden:

$$
f_{*}=\frac{n r}{n \cos \alpha-\cos \varphi-\frac{r}{e_{\star}}} \text { und } f \dagger=\frac{n r \cos ^{2} \alpha}{n \cos \alpha-\cos \varphi-\frac{r}{e_{\dagger}^{\dagger}} \cos ^{2} \varphi} .
$$

Da aber

$$
n \cos \alpha-\cos \varphi=\frac{\sin (\varphi-\alpha)}{\sin \alpha},
$$

so lassen sich die Hauptgleichungen auch folgendermassen schreiben :

$$
\left\{\begin{array}{l}
\frac{\sin \alpha}{e_{*}}+\frac{\sin \varphi}{f_{*}}=\frac{\sin (\varphi-\alpha)}{r} \\
\text { und } \frac{\sin \alpha \cos ^{2} \varphi}{e^{\dagger}}+\frac{\sin \varphi \cos ^{2} \alpha}{f^{\dagger}}=\frac{\sin (\varphi-\alpha)}{r} \text {. }
\end{array}\right.
$$

Hieraus leitet sich ab

$$
\text { für } e *=e \dot{\dagger}=e \text { : }
$$

(2) $\frac{\cos ^{2} \alpha}{f_{\dagger}^{\dagger}}-\frac{\cos ^{2} \varphi}{f_{\star}}=\frac{\sin \varphi \sin (\varphi-\alpha)}{r}$ und $\frac{\cos ^{2} \alpha}{f_{\dagger}}-\frac{1}{f_{\star}}=\frac{\sin \varphi \sin \alpha}{e}$;

$$
\text { für } f_{*}=f \dagger=f \text { : }
$$

(3) $\frac{\cos ^{2} \varphi}{e_{\dagger}}-\frac{\cos ^{2} \alpha}{e_{*}}=\frac{\sin \alpha \sin (\varphi-\alpha)}{r}$ und $\frac{\cos ^{2} \varphi}{e_{\dagger}^{\dagger}}-\frac{1}{e_{*}}=\frac{\sin \varphi \sin \alpha}{f}$;

$$
\text { für } \mathrm{e}=\infty \text { : }
$$

$$
F_{*}^{*}=r \frac{\sin \varphi}{\sin (\varphi-\alpha)} \text { und } F_{\dagger} \dagger=r \frac{\sin \varphi \cos ^{2} \alpha}{\sin (\varphi-\alpha)}
$$

für $f=\infty$ :

$E_{*}=r \frac{\sin \alpha}{\sin (\varphi-\alpha)}$ und $E \dagger=r \frac{\sin \alpha \cos ^{2} \varphi}{\sin (\varphi-\alpha)}$ 
Brechung bei schiefer Incidenz, mit besonderer Berücksichtigung d. Auges. 293 woraus folgt:

$$
\frac{E^{*}}{e_{*}}+\frac{F^{*}}{f_{*}}=1 \text { und } \frac{E_{\dagger}}{e_{\dagger}}+\frac{F_{\dagger}}{f \dagger}=1
$$

c. Ausdrücke für eine Linse vom Index $n$.

An der vorderen Fläche seien die Bezeichnungen wie bisher, die hintere habe den Radius $\varrho$, die Winkel $\beta$ innen und $\psi$ aussen; die Dicke der Linse sei $d$. Setzt man das in der Linse verlaufende Stïck des Strahlenbündels $=T$, so ist

$$
T=r \cos \alpha+\varrho \cos \beta-\sqrt{(r+\varrho-d)^{2}-(r \sin \alpha+\varrho \sin \beta)^{2}} .
$$

Nennt man $e$ und $f$ die Entfernungen zweier conjugirter Punkte von den Linsenflächen and $e^{\prime}$ und $f^{\prime}$ die Abstände ihrer durch die nächste Linsenfläche gelieferten Bilder von dieser Fläche, so muss sein

$$
e^{\prime}+f^{\prime}=T
$$

Die Werthe $e^{t}$ und $f^{t}$ ergeben sich aus den Gleichungen 1, unter Berücksichtigung der nöthigen Einsetzungen, und man erhält

$$
\left\{\begin{aligned}
\text { und } & \left(\frac{A}{r}-\frac{1}{e_{*}}\right)\left(\frac{B}{\varrho}-\frac{1}{f_{*}}\right)=\frac{n}{T}\left(\frac{A}{r}-\frac{1}{e^{2} \varphi}+\frac{B}{\varrho}-\frac{1}{f_{*}}\right) \\
& =\frac{n}{T}\left[\cos ^{2} \beta\left(\frac{A}{r}-\frac{\cos ^{2} \psi}{f^{\dagger} \dagger}\right)\right.
\end{aligned}\right.
$$

worin $A=\frac{\sin (\varphi-\alpha)}{\sin \alpha}$ und $B=\frac{\sin (\psi-\beta)}{\sin \beta}$.

Aus diesen Gleichungen (identisch mit den Gl. 26, 27 d. II. Theils) lassen sich leicht die Werthe von $E *, E \dagger, F \star, F \dagger$ ableiten.

d. Ausdrücke für Durchgang durch den optischen Mittelpunkt.

Hier wird $\alpha=\beta, \varphi=\psi, A=B$ und

$$
T=(r+\varrho) \cos \alpha-\sqrt{D^{2}-(r+\varrho)^{2} \sin ^{2} \alpha}, \quad(D=r+\varrho-d),
$$

wodureh sich die Gleichungen 7 sehr vereinfachen ( $\nabla$ gl. Gl. 35 und 36 des II. Theils).

Schon im II. Theil wurde die Bemessung der Bildorte von den Bildern des optischen Mittelpunktes aus eingefuihrt, durch welche die Ausdritcke besonders einfache Formen an- 
nehmen. Der optische Sinn dieser Bemessung ist folgender: Damit die von zwei Punkten $a$ und $b$ ausgehenden Strahlen nach der Brechung an der ersten Linsenfläche durch den optischen Mittelpunkt $c$ gehen, dürfen sie nicht auf einen und denselben Punkt der vorderen Fläche gerichtet sein, sondern sie müssen gerichtet sein auf das Bild $c^{t}$, welches die vordere Fläche vom Punkte $c$ entwirft. Da also $c^{\prime}$ der geforderte gemeinsame Punkt der einfallenden Strahlen ist, so ist auch $c^{\prime} a$ und $c^{\prime} b$ das richtige Distanzmass der Objecte $a$ und $b$, und ebenso $c^{\prime \prime} \alpha$ und $c^{\prime \prime} \beta$ das richtige Mass der Bilddistanz, wenn $c^{\prime \prime}$ das Bild von $c$ durch die hintere Linsenfläche, und $\alpha$ und $\beta$ die Bilder von $a$ und $b$ sind. Denn offenbar wird $a b: \alpha \beta=c^{a} a: c^{\prime \prime} \alpha$ sich verhalten. Die Punkte $c^{\prime}$ und $c^{\prime \prime}$ spielen also für den schiefen Durchgang dieselbe Rolle, wie die Hauptpunkte der Linse für den normalen Durchgang. Es giebt aber für jede Incidenzschiefe zwe i Paare solcher Cardinalpunkte, nämlich $c^{*}, c^{\prime \prime}{ }_{*}$ und $c^{\prime} \uparrow$ und $c^{\prime \prime} \uparrow$ (in den früheren Theilen mit $\varphi_{2}, \psi_{2}$ und $\varphi_{1}, \psi_{1}$ bezeichnet). Von diesen Punkten ans gerechnet, ergeben sich als Hauptbrennweiten der Linse

(8) $\left\{\begin{array}{l}E^{*}=F_{*}=\frac{r \varrho \sin \varphi}{P_{*} \sin (\varphi-\alpha)}, \text { worin } P_{*}=n(r+\varrho)-\frac{\sin (\varphi-\alpha)}{\sin \alpha} T \\ E_{\dagger}=F^{\dagger}=\frac{r \varrho \sin \varphi \cos ^{2} \varphi \cos ^{2} \alpha}{P \dagger \sin (\varphi-\alpha)}, P_{\dagger}=n(r+\varrho) \cos ^{2} \alpha-\frac{\sin (\varphi-\alpha)}{\sin \alpha} T\end{array}\right.$ Nennt man $\varepsilon$ den In c i d e n z w i n k e l, d. h. den Winkel zwischen dem einfallenden Strahl und der Axe, ferner $\vartheta$ den Winkel zwischen dem durchgehenden gebrochenen Strahl and der Axe, so ergeben sich aus der geometrischen Betrachtung (s. Fig. 6 im II. Theil sowie unten Fig. 1) folgende Beziehungen:

$$
\begin{gathered}
\varepsilon=\vartheta+\varphi-\alpha \\
(r+\varrho) \sin \alpha=D \sin \vartheta \\
T=(r+\varrho) \cos \alpha-D \cos \vartheta=D \frac{\sin (\vartheta-\alpha)}{\sin \alpha}=(r+\varrho) \frac{\sin (\vartheta-\alpha)}{\sin \vartheta} \\
P_{\star}=D \frac{\sin \varepsilon}{\sin \alpha}=(r+\varrho) \frac{\sin \varepsilon}{\sin \vartheta} \\
P \dagger=D\left(\frac{\sin \varepsilon}{\sin \alpha}-\sin \vartheta \sin \varphi\right)=(r+\varrho)\left(\frac{\sin \varepsilon}{\sin \vartheta}-\sin \varphi \sin \alpha\right),
\end{gathered}
$$

so dass

$$
\left\{\begin{array}{l}
\frac{1}{E_{*}}=\frac{1}{F_{*}}=\left(\frac{1}{r}+\frac{1}{\varrho}\right) \frac{\sin \varepsilon \sin (\varphi-\alpha)}{\sin \vartheta \sin \varphi} \\
\frac{1}{E_{\dagger} \dagger}=\frac{1}{F \dagger}=\left(\frac{1}{r}+\frac{1}{\varrho}\right) \frac{(\sin \varepsilon-\sin \vartheta \sin \varphi \sin \alpha) \sin (\varphi-\alpha)}{\sin \vartheta \sin \varphi \cos ^{2} \varphi \cos ^{2} \alpha} .
\end{array}\right.
$$


Der Abstand der Bilder des optisehen Mittelpunktes von den zugehörigen Flächen (d. h. der „Directions"- und der „Seitenpunete“) ist

$$
\varphi_{*}=\frac{r T}{P_{\star}}, \quad \varphi \dagger=\frac{r T \cos ^{2} \varphi}{P \dagger}, \quad \psi^{*}=\frac{\varrho T}{P_{\star}}, \quad \psi_{\dagger}=\frac{\varrho T \cos ^{2} \varphi}{P \dagger} .
$$

Der gegenseitige Abstand der beiden Directionspuncte ist

$$
\Delta_{*}=D \frac{\sin (\vartheta-\alpha) \sin (\varphi-\alpha)}{\sin \varepsilon \sin \alpha}=(r+\varrho)\left(\frac{\sin \varphi}{\sin \varepsilon}-\frac{\sin \alpha}{\sin \vartheta}\right),
$$

und der gegenseitige Abstand der beiden Seitenpuncte

$$
\Delta \dagger=\frac{D}{\sin \alpha} \frac{\sin \vartheta \sin \varphi-\sin \varepsilon \sin \alpha}{\sin \varepsilon-\sin \vartheta \sin \varphi \sin \alpha} \sqrt{\sin ^{2}(\vartheta+\varphi)+\cos ^{2} \vartheta \cos ^{2} \varphi}
$$

Zahlreiche andere Beziehungen, welche sich aufstellen lassen, unterlasse ich hier anzuführen.

e. Die Periscopie bei Durchgang durch den optischen Mittelpunkt.

\section{1) Der Ausdrnck für die Bildgüte.}

Die Bildgüte $B$ ist, wie schon im II. Theile angeführt, zu bemessen nach der Differenz der Bildgrössen, bezogen auf ihre absolute Grösse, so dass, da die Bildgrössen den Distanzen von den Cardinalpunkten proportional sind,

$$
\frac{1}{B}=\frac{f_{*}-f_{\dagger}^{\dagger}}{f^{*}}
$$

Für unendlich entfernte Objecte ergiebt sich durch Einsetzung von Gl. 9

$$
\frac{1}{B}=1-\frac{\sin \varepsilon \cos ^{2} \varphi \cos ^{2} \alpha}{\sin \varepsilon-\sin \vartheta \sin \varphi \sin \alpha} .
$$

Zu dem gleichen Werthe gelangt man, wie schon im II. Theil gezeigt worden ist, noch auf einem anderen Wege. Nennt man nämlich $G *$ und $G \dagger$ die Entfernungen der vorderen Brennlinien der ersten Linsenfläche von den zugehörigen Cardinalpunkten, so dass $G_{*}=E^{*}+\varphi *$ ete. ( $E^{*}$ aus Gl. 5 entnommen), so ergiebt sich $G *: G \dagger=F *: F \dagger$

so dass man auch setzen kann

$$
\frac{1}{B}=1-\frac{G \dagger}{G *}
$$

Für die Bildgïte kommt also der Werth von $\varrho$ nicht in Betracht; es genügt (Durchgang durch den optischen Mittelpunkt vorausge- 
setzt) die Kenntniss des einen Linsenabschnittes, z. B. des vorderen Krimmungsradius und des vorderen Theils der Linsendicke (bis zum opt. Mittelpunkt). Dieser wichtige Satz ist allgemeingültig und erleichtert die Berechnung für zusammengesetzì Linsen, welche einen optischen Mittelpunkt haben, bedeutend (s. unten).

Der eben angeführte Satz hat seinen tieferen Grund in der ganz allgemeinen, auch für den normalen Durchgang geltenden Beziehung, dass der reciproke Werth der Brennweite einer Linse gleich ist der Summe der reciproken Werthe der Entfernungen zwischen den äusseren Einzelbrennpunkten der Linsenflächen und den zugehörigen Hauptpunkten; bezeichnet man diese Entfernungen mit $G$ und $H$, so ist

$$
\frac{1}{F}=\frac{1}{G}+\frac{1}{H}
$$

und ebenso für schiefen Durchgang

$$
\begin{aligned}
& \frac{1}{F_{\star}}=\frac{1}{G^{*}}+\frac{1}{H_{\star}} \\
& \frac{1}{F_{\dagger}^{\prime}}=\frac{1}{G_{\dagger}}+\frac{1}{H_{\dagger}^{*}}
\end{aligned}
$$

Da sich ferner $G: H$ verhält wie $r: \varrho$, und ebenso $G^{\star}: H \star$ und $G \dagger: H \dagger$, so folgt $G^{*}: G \dagger=F_{\star}: F \dagger$.

\section{2) Die corrigirende Cylinderlinse.}

Als den der Wahrheit nächsten Ausdruck für die Kraft einer corrigirenden Cylinderlinse haben wir im II. Theil angenommen

$$
\frac{1}{\Phi}=\frac{1}{F \dagger}-\frac{1}{F *},
$$

dabei aber bemerkt, dass diese Linse eigentlich nicht existirt, da die verglichenen Brennweiten von verschiedenen Punkten aus zählen, nämlich von den beiden Cardinalpunkten $\varphi^{*}$ und $\varphi \dagger$ (oder $\psi *$ and $\psi \dagger$ ).

Es ist mir seitdem gelungen, einen vollkommen exacten Ausdruck für ein corrigirendes Cylinderglas zu bestimmen, welcher seine theoretische Strenge u. A. dadurch $z \mathfrak{u}$ erkennen giebt, dass er nicht bloss für die Correction bei unendlicher Entfernung des Objectes, sondern für jede beliebige Objectdistanz gültig bleibt, was bei dem obigen Ausdruck nur annähernd der Fall war (vgl. Theil II, p. 384). 
Das durch den optischen Mittelpunkt $e$ (Fig. 1) gehende Bündel

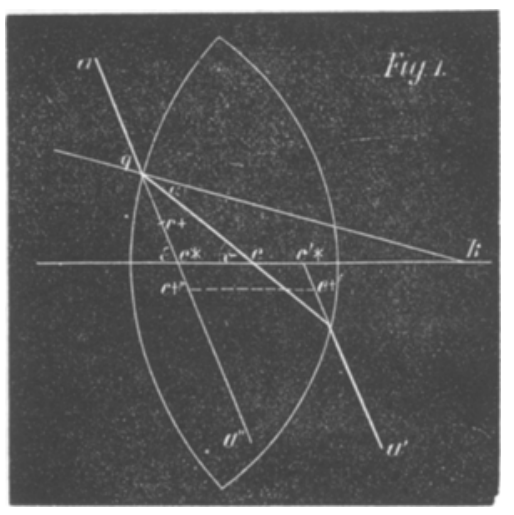
wird parallel mit sich selbst um eine gewisse Grösse $(\Sigma=$ $A * \sin \varepsilon)$ verschoben. Denken wir uns diese Verschiebung beseitigt, d. h. den gebrochenen Strahl $c *^{\prime} a^{\prime}$ in die Verlängerung des einfallenden, nach $c * a^{\prime \prime}$ verlegt, so lässt sich die Wirkung des Systems anf die zweier Linsen reduciren, welche zu $a a^{\prime \prime}$ normal stehen. Die Hauptschnittbrechung erfolgt durch eine unendlich dünne Linse, welche nur Einen Hauptpunkt, und zwar im Directionspunkt $c$ * hat, die Kreuzschnittbrechung dagegen durch eine Linse mit den zwei Hauptpunkten $c \nmid$ und $c \dagger^{\prime \prime}$. Das corrigirende Glas ist also so zu wählen, wie für eine Linse mit den beiden Hauptpunkten $c \nmid$ und $c \dagger^{\prime \prime}$ und der Brennweite $F \dagger$, und eine zweite Linse mit der Brennweite $F^{*}$, deren Hauptpunkt $c^{*}$ in den optischen Mittelpunkt der ersten Linse fällt; die Kraft der zweiten Linse ist mit negativem Vorzeichen zu nehmen.

Zur Lösung dieser Aufgabe habe ich folgenden Weg eingeschlagen:

1. Wir substituiren für die dicke Linse mit den Hauptpunkten

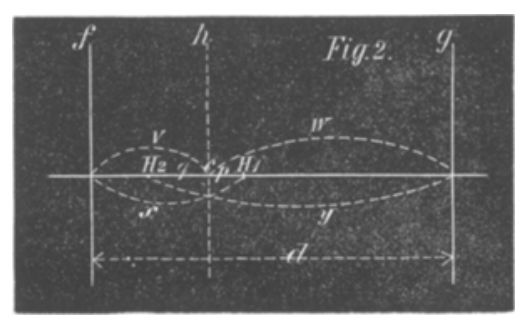
$c \dagger$ nnd $c \dagger^{\prime \prime}$ zwei unendlich dünne Linsen (Fig. 2), jedoch so, dass das System dieselben Cardinalpunkte behält. Nennen wir $\lambda$ die Brennweite der dicken Linse, $p, q$ die Distanzen ihrer Hauptpunkte vom optischen Mittelpunkt ( $c * c \dagger$ und $\left.c * c \dagger^{\prime \prime}\right)$, ferner $f, g, d$ Brennweiten und gegenseitigen Abstand der substituirten Linsen, endlich $x$ und $y$ deren Abstand von den (negativ liegenden) Hauptpunkten, so erhalten wir die Bedingungsgleichungen

$$
\begin{aligned}
& d=x-p+y-q=f+g-\frac{f g}{\lambda} \\
& x: y=p: q \\
& \frac{1}{x-p}-\frac{1}{x}=\frac{1}{f} \quad \text { und } \quad \frac{1}{y-q}-\frac{1}{y}=\frac{1}{g},
\end{aligned}
$$


worans sich ergiebt

$$
\begin{aligned}
& f=\frac{1}{q}[(p+q) \lambda-\sqrt{p q(p+q) \lambda}] \\
& g=\frac{1}{p}[(p+q) \lambda-\sqrt{p q(p+q) \lambda}] \\
& d=(p+q)\left(\sqrt{\left.\left(\frac{1}{p}+\frac{1}{q}\right) \lambda-1\right)}\right.
\end{aligned}
$$

2. Jetzt werde eine dritte Linse von der Brennweite $h$ in den optischen Mittelpunkt des Systems $f, g, d$ gestellt und die Cardinalpunkte des ganzen Systems der drei Linsen gesucht. Die Entfernungen der mittleren linse von den beiden äusseren mögen $\operatorname{mit}^{\prime \prime} v$ und $w$ bezeichnet werden, so dass

$$
v=\frac{d f}{f+g}, w=\frac{d g}{f+g} .
$$

Verfolgt man die Abbildung eines Punktes von gegebener Lage successive durch die drei Flächen hindurch, so kann man die Lage der Brenn- und Hauptpunkte des Systems leicht nach bekannten Principien berechnen, und erhält als Brennweite

$$
F^{\prime}=\frac{f g h}{f g+h(f+g)+v w-v(g+h)-w(f+h)},
$$

and mit Einsetzung der Werthe von $v, w$ und der Brennweite $\lambda$ des Systems $f, g$ für sich:

$$
\frac{1}{F}=\frac{1}{\lambda}+\frac{1}{h}\left(\frac{f+g-a}{f+g}\right)^{2}=\frac{1}{\lambda}+\frac{1}{h}\left(\frac{\frac{1}{\lambda}}{\frac{1}{f}+\frac{1}{g}}\right)^{2} .
$$

Als Hauptpunktdistanz ergiebt sich

$$
H=\frac{d^{2}}{d-f-g}
$$

d. h. die Lage der Hauptpunkte wird, wie vorauszusehen war, durch die Einfiugung der dïnnen Linse $h$ in den optisehen Mittelpunkt nicht verändert.

3. Nun endlich müssen für $f, g, d$ ihre oben gefundenen Werthe eingesetzt werden, so dass sich ergiebt

$$
\frac{1}{F}=\frac{1}{\lambda}+\frac{1}{h}\left[1-\sqrt{\frac{p q}{(p+q) \lambda}}\right]^{2}=\frac{1}{\lambda}+\frac{1}{h}\left[1-\sqrt{\frac{\frac{1}{\lambda}}{\frac{1}{p}+\frac{1}{q}}}\right]^{2} .
$$


Weiter ist, um unsere Aufgabe zu lösen, einzusetzen: $F \dagger$ für $\lambda$, $-F_{*}$ für $h, c * c \dagger=\varphi^{*}-\varphi \dagger$ für $p$, und $c_{*} c \dagger^{\prime \prime}=\psi *-\psi \dagger$ für $q$, so dass

$$
\frac{1}{\Phi}=\frac{1}{F_{\dagger}}-\frac{1}{F_{*}}\left[1-\sqrt{\frac{\frac{1}{F_{\dagger}}}{\frac{1}{\varphi^{*}-\varphi_{\dagger}^{\dagger}}+\frac{1}{\psi^{*}-\psi_{\dagger}^{\dagger}}}}\right]^{2}
$$

den völlig strengen und für jede Objectdistanz gültigen Ausdruck für die Kraft des in $c *$ zu legenden corrigirenden Cylinderglases darstellt.

Setzat man für $F^{*}, F \dot{\dagger}, \varphi^{*}$ etc. ihre oben gefundenen Werthe ein, so erhält man nach vielfachen Umformungen

$$
\begin{gathered}
\frac{1}{\Phi}=\left(\frac{1}{r}+\frac{1}{\varrho}\right) \frac{\sin \alpha}{\sin \vartheta}(\operatorname{tg} \varphi-\operatorname{tg} \alpha)\left\{\sin \varepsilon\left(1+\frac{\operatorname{tg} \varphi \operatorname{tg} \alpha}{\operatorname{tg} \vartheta}\right)\right. \\
\left.+2(\operatorname{cotg} \alpha-\operatorname{cotg} \varphi) \sqrt{\sin \varepsilon\left(\sin \varepsilon-\frac{\sin \varphi \sin \alpha}{\sin \vartheta}\right)}\right\}
\end{gathered}
$$

als strengen und allgemeingültigen Werth für die Astigmasie bei schiefem Durchgang durch die Mitte einer dicken Linse.

\section{Schiefer Durchgang durch geschichtete Linsen.}

Wir beschränken uns auf solche Linsen, welche einen optischen Mittelpunkt haben und auf Durchgang durch diesen Mittelpunkt. Ein optischer Mittelpunkt, d. h. ein Punkt von der Eigenschaft, dass jeder durch ihn gehende Strahl vor und nach der Brechung gleiche Richtung zur Axe hat, wird in dem Falle existiren, wo das vordere und das hintere Schichtensystem symmetrisch oder doch zu einem Punkte proportional angeordnet sind.

Nach dem oben S. 296 aufgestellten Satze ist es für unsere Aufgabe nurnöthig, die vorderen Brennweiten des vorderen Sehichtensystems für sich, bemessen von den zugehörigen vorderen Cardinalpunkten, zu bestimmen. Wir nehmen zunächst nur Eine, und zwar der äusseren Fläche (Radius $r$, Brechungsverhältniss $n$ ) concentrische Grenzfläche an, und bestimmen deren Einfluss. Thr Krümmungsradius (Fig. 3) sei $r^{\prime}$ und ihr Brechungsverhältniss $p$, so dass also der Index des Kerns $=n p$ ist. Der vordere Theil der Linsendicke, d. h. der Abstand des optischen Mittelpunktes von der vorderen Linsenfläche, sei $\delta$. 
Nennen wir die Winkel des Strahles mit dem Radius bei der ersten Brechung wie bisher $\varphi$ und $\alpha$, bei der zweiten $\varphi^{\prime}$ und $\alpha^{\prime}$, ferner $\varepsilon$ den Winkel des einfallenden Strahles mit der Axe und $\vartheta$ den des an beiden Flächen gebrochenen Strahles mit der Axe (am optischen Mittelpunkt), so haben wir aus dem Brechungsgesetz und aus der trigonometrischen Betrach-

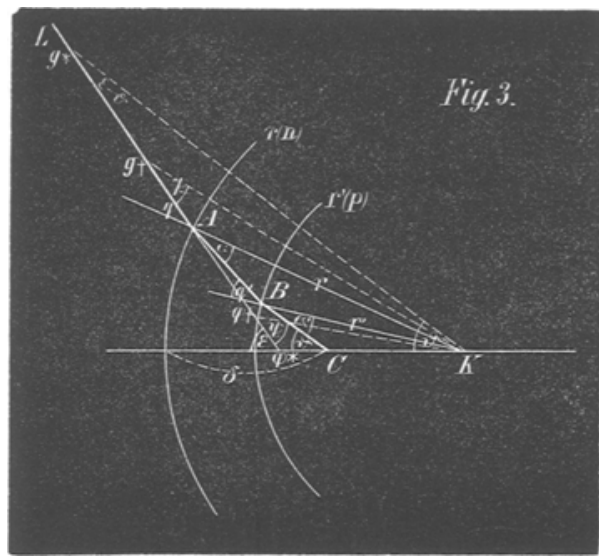
tung der Figur 3 folgende Beziehungen.

$$
\begin{gathered}
\sin \varphi=n \sin \alpha \\
r^{\prime} \sin \varphi^{\prime}=r \sin \alpha \\
\sin \varphi^{\prime}=p \sin \alpha^{\prime} \quad \text { oder } \quad p \sin \alpha^{\prime}=\frac{r}{r^{\prime}} \sin \alpha \\
(r-\delta) \sin \vartheta=r^{\prime} \sin \alpha^{\prime} \quad \text { oder } \quad p(r-\delta) \sin \vartheta=r \sin \alpha \\
\varepsilon=\vartheta+\varphi-\alpha+\varphi^{\prime}-\alpha^{\prime} .
\end{gathered}
$$

Um nun die zur Aufstellung der G1. 11 erforderlichen Grössen $G *$ und $G \dagger$ zu berechnen, dienen die Hauptgleichungen 1. Die vorderen Brennweiten des vorderen Systems für sich mögen, von der vorderen Linsenfläche aus bemessen, mit $g *$ und $g \dagger$ bezeichnet werden; es ist dann

$$
G *=g *-\varphi *, \quad G \dagger=g \dagger-\varphi \dagger,
$$

worin $\varphi^{*}, \varphi \dagger$ wie bisher die Entfernungen der vorderen Bilder des optischen Mittelpunktes von der vorderen Linsenfläche bedenten (werden diese Grössen absolut genommen, so müssen sie mit + Vorzeichen stehen; so wurde es in den vorigen Theilen bezeichnet).

Die Grössen $g_{*}, g \dagger, \varphi *, \varphi \dagger$ sind leicht nach demselben Verfahren zu finden, welches zur Auffindung der Gleichung 7 diente. Soll nämlich eine vordere Brennweite der ersten Fläche, z. B. $e$, conjugirt sein zu einer hinteren Brennweite der zweiten Fläche, z. B. $f^{\prime}$, so müssen die zu $e$ und $f^{\prime}$ gehörigen conjugirten Weiten $e^{\prime}$ und $f$ als algebraische Summe die Länge des Strahlenabschnittes zwischen beiden Flächen ergeben, $d . \mathrm{h}$. in unserem Falle die Länge $A B=r \cos \alpha-r^{\prime} \cos \varphi^{\prime}$. 
Brechung bei schiefer Incidenz, mit besonderer Berücksichtigung d. Auges. 301

Nun ist $g^{*}$ conjugirt $\mathrm{zu} f^{*}=\infty$

$$
\begin{array}{lll}
g \dagger & \quad " f^{\prime} \dagger=\infty \\
\varphi^{*} & \Rightarrow & \quad f^{\prime}=B C=r^{\prime} \cos \alpha^{\prime}-(r-\delta) \cos \vartheta \\
\varphi \dagger & \quad & f^{\prime} \dagger=B C=r^{\prime} \cos \alpha^{\prime}-(r-\delta) \cos \vartheta .
\end{array}
$$

Die Berechnung; beispielsweise für $g *$, gestaltet sich also folgendermassen: Setzt man in Gl. $1 f^{*}=\infty$, so erhält man

$$
e^{*}=\frac{r^{\prime} \sin \alpha^{4}}{\sin \left(\varphi^{\prime}-\alpha^{\prime}\right)}
$$

setzt man ferner $e^{*}=g^{*}$, so erhält man

$$
f^{*}=\frac{\sin \varphi}{\frac{\sin (\varphi-\alpha)}{r}-\frac{\sin \alpha}{g_{*}}}
$$

und da sein muss

so ergiebt sich

$$
f_{*}+e^{\prime}{ }_{*}=A B=r \cos \alpha-r^{\prime} \cos \varphi^{\prime},
$$

$$
\frac{r^{\prime} \sin \alpha^{\prime}}{\sin \left(\varphi^{\prime}-\alpha^{\prime}\right)}+\frac{\sin \varphi}{\frac{\sin (\varphi-\alpha)}{r}-\frac{\sin \alpha}{g^{\star}}}=r \cos \alpha-r^{\prime} \cos \varphi^{\prime},
$$

welche Gleichung für $g *$ za lösen ist. Ebenso erhält man auf dem angegebenen Wege für die drei anderen gesuchten Grössen die Bedingungsgleichungen:

$$
\begin{gathered}
\frac{r^{\prime} \sin \alpha^{\prime} \cos ^{2} \varphi^{\prime}}{\sin \left(\varphi^{\prime}-\alpha^{\prime}\right)}+\frac{\sin \varphi \cos ^{2} \alpha}{\frac{\sin (\varphi-\alpha)}{r}-\frac{\sin \alpha \cos ^{2} \varphi}{g \dagger}}=r \cos \alpha-r^{\prime} \cos \varphi^{\prime} . \\
\frac{\sin \alpha^{\prime}}{\sin \left(\varphi^{\prime}-\alpha^{\prime}\right)}-\frac{\sin \varphi^{\prime}}{r^{\prime}}-\frac{\sin \varphi}{r^{\prime} \cos \alpha^{\prime}-(r-\delta) \cos \vartheta} \\
=r \cos \alpha-r^{\prime} \cos \varphi^{\prime} \\
\frac{\sin (\varphi-\alpha)}{r}-\frac{\sin \alpha}{\varphi^{*}} \\
\frac{\sin \alpha^{\prime} \cos ^{2} \varphi^{\prime}}{\sin ^{\prime}\left(\varphi^{\prime}-\alpha^{\prime}\right)} \\
r^{\prime}-\frac{\sin \varphi^{\prime} \cos ^{2} \alpha^{\prime}}{r^{\prime} \cos \alpha^{\prime}-(r-\delta) \cos \vartheta} \\
=r \cos \alpha-r^{\prime} \cos \varphi^{\prime} .
\end{gathered}
$$

Löst man diese 4 Gleichungen fïr $g *, g \dagger, \varphi^{*}, \varphi \dagger$, so erbält man ziemlich complicirte Ausdrücke; dieselben vereinfachen sich aber schon beträchtlich, wenn man daraus die eigentlich gesuchten Grössen $G^{*}$ und $G \dagger$ bildet, noch mehr aber, wenn man für die Grössen $\frac{r^{\prime}}{r}, \frac{r-\delta}{r^{\prime}}$ u. s. w. die aus den Gleichungen 13-16 sich ergebenden Sinusquotienten einführt und ferner sich bemiiht, überall 
Tangenten einzuführen. So erhält man nach mannigfachen Umformungen die lehrreichen Gleichungen:

$$
\begin{aligned}
& G *=g^{*}-\varphi *=r \cdot \frac{\sin \vartheta \sin \varphi}{\sin \varepsilon \sin \left(\varphi-\alpha+\varphi^{\prime}-\alpha^{\prime}\right)} \\
& G \dagger=g \dagger-\varphi \dagger
\end{aligned}
$$

$=r \cdot \sin \varphi \frac{\operatorname{tg} \vartheta}{\left(\operatorname{tg} \varphi-\operatorname{tg} \alpha+\operatorname{tg} \varphi^{\prime}-\operatorname{tg} \alpha^{\prime}\right)\left(\operatorname{tg} \vartheta+\operatorname{tg} \varphi-\operatorname{tg} \alpha+\operatorname{tg} \varphi^{\prime}-\operatorname{tg} \alpha^{\prime}\right)}$

Setzt man hierin

$$
\begin{gathered}
\operatorname{tg} \varphi-\operatorname{tg} \alpha+\operatorname{tg} \varphi^{\prime}-\operatorname{tg} \alpha^{\prime}=\operatorname{tg} \chi . \\
\operatorname{tg} \vartheta+\operatorname{tg} \chi=\operatorname{tg} \psi,
\end{gathered}
$$

und berïcksichtigt man, dass $\varepsilon=9+\varphi-\alpha+\varphi^{\prime}-\alpha^{\prime}$, also $\sin \left(\varphi-\alpha+\varphi^{\prime}-\alpha^{\prime}\right)=\sin (\varepsilon-\vartheta)$, so ergeben sich die ungemein einfachen Gleichungen

$$
\begin{gathered}
G^{*}=r \cdot \frac{\sin \varphi}{\sin \varepsilon \cos \varepsilon} \cdot \frac{\operatorname{tg} \vartheta}{\operatorname{tg} \varepsilon-\operatorname{tg} \vartheta} \\
G+=r \cdot \sin \varphi \cdot \frac{\operatorname{tg} \vartheta}{\operatorname{tg} \psi \operatorname{tg} \psi}
\end{gathered}
$$

also für die Bildgüte

$$
\frac{1}{B}=1-\sin \varepsilon \cos \varepsilon \frac{\operatorname{tg} \varepsilon-\operatorname{tg} \vartheta}{\operatorname{tg} \chi \operatorname{tg} \psi}
$$

oder, da $\operatorname{tg} \vartheta=\operatorname{tg} \psi-\operatorname{tg} \psi$,

(29) $\frac{1}{B}=1-\sin \varepsilon \cos \varepsilon(\operatorname{cotg} \psi-\operatorname{cotg} x+\operatorname{tg} \varepsilon \operatorname{cotg} \psi \operatorname{cotg} x)$.

Diese völlig strenge und dabei überraschend einfache Gleichung für die Bildgüte einer concentrisch geschichteten Linse erweist sich zugleich als völlig allgemeingültig, wie viele Schichten man auch annehmen möge; denn aus dem Ausdruck für tg $\chi$ ergiebt sich, dass derselbe, wenn noch eine dritte Grenzschicht mit den Winkeln $\varphi^{\prime \prime}, \alpha^{\prime \prime}$ und noch weitere hinzukommen, die Form behält (30) $\operatorname{tg} \chi=\operatorname{tg} \varphi-\operatorname{tg} \alpha+\operatorname{tg} \varphi^{\prime}-\operatorname{tg} \alpha^{\prime}+\operatorname{tg} \varphi^{\prime \prime}-\operatorname{tg} \alpha^{\prime \prime}+\ldots=\Sigma(\operatorname{tg} \varphi-\operatorname{tg} \alpha)$ und ebenso

$$
\operatorname{tg} \psi=\operatorname{tg} \vartheta+\Sigma(\operatorname{tg} \varphi-\operatorname{tg} \alpha) .
$$

Auch kann man sich leicht tiberzergen, dass die fuir die homogene Linse gefundene Gleichung 10 mit der Gleichung 29 identisch wird, wenn man setzt $\operatorname{tg} \chi=\operatorname{tg} \varphi-\operatorname{tg} \alpha, \operatorname{tg} \psi=\operatorname{tg} \vartheta+\operatorname{tg} \varphi-\operatorname{tg} \alpha$.

Die eingefiubrten Winkel $\chi$ und $\psi$ haben aber eine leicht definirbare geometrische Bedeutung. Zieht man nämlich vom Krümmungsmittelpankt $K$ (Fig. 8 ) gerade Linien zu den Punkten $g_{*}, g_{\dagger}$ and $\varphi \dagger$ und bestimmt die Winkel, welche dieselben mit 
Brechung bei schiefer Incidenz, mit besonderer Berüicksichtignng d. Auges. 303

dem einfallenden Strahl $L \varphi_{*}$ bilden, so ergiebt sich aus dem Dreieck $A g \dagger K$ (worin $A g \dagger=g \dagger$ ):

$$
\sin A g \dagger K: \sin (\varphi-A g \dagger K)=r: g \dagger
$$

und durch Einsetzung des aus Gl. 19 abgeleiteten Werthes von $g \dagger$ findet man

also

$$
\operatorname{tg} A g \dagger K=\operatorname{tg} \varphi-\operatorname{tg} \alpha+\operatorname{tg} \varphi^{\prime}-\operatorname{tg} \alpha^{\prime}
$$

Winkel $A g \dagger K=\chi$.

In gleicher Weise ergiebt sich aus dem Dreieck $A \varphi \dagger K$, worin Acpt $=\varphi \dagger$,

$$
\sin \varphi_{*} \varphi \dagger K: \sin \left(\varphi^{*} \varphi \dagger K-\varphi\right)=r: \varphi \dagger
$$

und durch Einsetzung des Werthes von $\varphi \dagger$ (aus Gl. 21)

also

$$
\operatorname{tg} \varphi^{\star} \varphi \dagger K=\operatorname{tg} \vartheta+\operatorname{tg} \varphi-\operatorname{tg} \alpha+\operatorname{tg} \varphi^{\prime}-\operatorname{tg} \alpha^{\prime}
$$

\section{Winkel $\varphi^{*} \varphi \dagger K=\psi$.}

Die drei in der Gleichung 29 vorkommenden Winkel sind also einfach die Winkel, welche der einfallende Strahl an den Cardinalpunkten mit den zum Krümmungsmittelpunkt gehenden Graden einschliesst, and die Tangenten dieser Winkel stehen zu den Winkeln $\varphi, \alpha, \varphi^{\prime}, \alpha^{\prime}$ etc. und $\vartheta$ in der durch Gleichung 30 und 31 ausgedruickten einfachen Beziehung.

Der Winkel bei $g *$ ist ebenfalls leicht anszudrücken. Aus der Eigenschaft des Punktes $g *$ folgt nämlich direct, dass $K g$ * parallel sein muss mit $C B$; also ist Winkel $g * K C=\vartheta$ und Winkel

$$
A g * K=\varepsilon-\vartheta
$$

Mittels dieser Winkelbestimmungen lassen sich auch die Grössen $G *=\varphi * g *$ und $G+=\varphi \dagger g \dagger$ direct aus den Dreiecken $g * \varphi^{*} \mathcal{K}$ und $g \dagger \varphi \dagger K$ ableiten, und man erhält so die Gleichungen 26 und 27.

Schliesslich soll noch die centrale vordere Partialbrennweite $G$ für geschichtete Linsen aufgestellt werden. Man erhält sie am einfachsten, wenn man in Gleichung 22 oder 23 die Winkel bis zum Verschwinden kleiner werden lässt; man erhält so

$$
G=n r \cdot \frac{\frac{1}{p} \cdot \frac{r}{r-\delta}}{\left[n-1+\frac{p-1}{p} \cdot \frac{r}{r^{\prime}}\right]\left[\frac{1}{p} \cdot \frac{r}{r-\delta}+n-1+\frac{p-1}{p} \cdot \frac{r}{r}\right]}
$$

oder

(33) $\frac{1}{G}=\frac{1}{n r}\left[n-1+\frac{p-1}{p} \frac{r}{r^{4}}+\frac{p(r-d)}{r}\left(n-1+\frac{p-1}{p} \frac{r}{r^{4}}\right)^{2}\right]$. 


\section{Der Einfluss der (concentrischen) Schichtung auf die Periscopie.}

Die Frage ist hier so zu stellen, $o b$ von zwei Linsen gleicher centraler Brennweite $(G)$ und gleicher äusserer Form $(r, \delta)$, bei gegebener Incidenzschiefe $(\varepsilon)$ die homogene oder die in gewisser Weise geschichtete Linse ein besseres Bild, d. h. einen höheren Werth von $B$ (Gl. 29), liefert.

Legt man eine Trennungsschicht vom Radius $r^{\prime}$ und dem Brechungsverhältniss $p$ in die Linse, so geht dieselbe in die homogene Linse über, wenn $p=1$ wird; der Kern ist ferner stärker oder schwächer brechend als die Hulle, je nachdem $p$ grösser oder kleiner als 1 ist. Man kann nun also die Frage mathematisch so formuliren: Wie ändert sich bei gegebenem $G, r, \delta, \varepsilon$ der Werth von $B$ mit Aenderung von $p$ ? Mit andern Worten, es ist unter den angegebenen Voraussetzungen der Werth $\frac{\partial B}{\partial p}$ aufzusuchen und sein Vorzeichen zu bestimmen. Da aber in Gl. $29 B$ um so grösser wird, je grösser der in Klammer stehende Ausdruck

$$
\operatorname{cotg} \psi-\operatorname{cotg} \chi+\operatorname{tg} \varepsilon \operatorname{cotg} \psi \operatorname{cotg} \chi=C,
$$

so genügt es, das Vorzeichen von

$$
\frac{\partial C}{\partial p}
$$

zu untersuchen, da $\frac{\partial B}{\partial p}$ dasselbe Vorzeichen haben muss.

Da $\varepsilon$ constant ist, so ist

$$
\frac{\partial C}{\partial p}=\frac{\partial C}{\partial \psi} \cdot \frac{\partial \psi}{\partial p}+\frac{\partial C}{\partial \chi} \cdot \frac{\partial \chi}{\partial p}=-\frac{\operatorname{tg} \varepsilon+\operatorname{tg} \chi}{\sin ^{2} \psi \operatorname{tg} \chi} \frac{\partial \psi}{\partial p}+\frac{\operatorname{tg} \psi-\operatorname{tg} \varepsilon}{\sin ^{2} \chi \operatorname{tg} \psi} \frac{\partial \chi}{\partial p} .
$$

Die Werthe von $\frac{\partial \psi}{\partial p}$ und $\frac{\partial \chi}{\partial p}$ sind ans den Gleichungen 30 und 31 zu ermitteln; es ist nämlich

$$
\begin{aligned}
\frac{\frac{\partial \psi}{\partial p}}{\cos ^{2} \psi} & =\frac{\frac{\partial \vartheta}{\partial p}}{\cos ^{2} \vartheta}+\frac{\frac{\partial p}{\partial p}}{\cos ^{2} \varphi}-\frac{\frac{\partial \alpha}{\partial p}}{\cos ^{2} \alpha}+\frac{\frac{\partial \varphi^{\prime}}{\partial p}}{\cos ^{2} \varphi^{\prime}}-\frac{\frac{\partial \alpha^{\prime}}{\partial p}}{\cos ^{2} \alpha^{\prime}}, \\
\text { und } \frac{\frac{\partial \psi}{\partial p}}{\cos ^{2} \psi} & =\frac{\frac{\partial \varphi}{\partial p}}{\cos ^{2} \varphi}-\frac{\frac{\partial \alpha}{\partial p}}{\cos ^{2} \alpha}+\frac{\frac{\partial \varphi^{\prime}}{\partial p}}{\cos ^{2} \varphi^{\prime}}-\frac{\frac{\partial \alpha^{\prime}}{\partial p}}{\cos ^{2} \ell^{\prime}}
\end{aligned}
$$

Die Differentialquotienten der Winkel $\vartheta, \varphi, \alpha$ ete. ergeben sich aus deren Beziehungen: Es folgt 
Brechung bei schiefer Incidenz, mit besonderer Berücksichtigung d. Auges. 305

aus Gl. 13: $\quad \cos \varphi \frac{\partial \varphi}{\partial p}=n \cos \alpha \frac{\partial \alpha}{\partial p}+\sin \alpha \frac{\partial n}{\partial p}$

aus Gl. 14: $\quad r^{\prime} \cos \varphi^{\prime} \frac{\partial \varphi^{\prime}}{\partial p}=r \cos \alpha \frac{\partial \alpha}{\partial p}$

aus Gl. 15: $\quad p \cos \alpha^{\prime} \frac{\partial \alpha^{\prime}}{\partial p}+\sin \alpha^{\prime}=\frac{r}{r^{\prime}} \cos \alpha \frac{\partial \alpha}{\partial p}$

aus Gl. 16: $\quad p(r-\delta) \cos \vartheta \frac{\partial \vartheta}{\partial p}+(r-\delta) \sin \vartheta=r \cos \alpha \frac{\partial \alpha}{\partial p}$

aus Gl. 17: $\left.\frac{\partial \varepsilon}{\partial p}=0^{1}\right)=\frac{\partial \vartheta}{\partial p}+\frac{\partial \varphi}{\partial p}-\frac{\partial \alpha}{\partial p}+\frac{\partial \varphi^{\prime}}{\partial p}-\frac{\partial \alpha^{\prime}}{\partial p}$.

Aus diesen 5 Gleichungen lassen sich die 5 Werthe bestimmen, indem man für $\frac{r}{r^{\prime}}$ etc. immer wieder die Sinusquotienten einführt :

$$
\begin{aligned}
& \frac{\partial \varphi}{\partial p}=\frac{\operatorname{tg} \varphi}{\operatorname{tg} \psi}\left(\frac{\operatorname{tg} \vartheta-\operatorname{tg} \alpha^{\prime}}{p}+\frac{\operatorname{tg} \psi-\operatorname{tg} \varphi}{n} \frac{\partial n}{\partial p}\right) \\
& \frac{\partial \alpha}{\partial p}=\frac{\operatorname{tg} \alpha}{\operatorname{tg} \psi}\left(\frac{\operatorname{tg} \vartheta-\operatorname{tg} \alpha^{\prime}}{p}-\frac{\operatorname{tg} \varphi}{n} \frac{\partial n}{\partial p}\right) \\
& \frac{\partial \varphi^{\prime}}{\partial p}=\frac{\operatorname{tg} \varphi^{\prime}}{\operatorname{tg} \psi}\left(\frac{\operatorname{tg} \vartheta-\operatorname{tg} \alpha^{\prime}}{p}-\frac{\operatorname{tg} \varphi}{n} \frac{\partial n}{\partial p}\right) \\
& \frac{\partial \alpha^{\prime}}{\partial p}=-\frac{\operatorname{tg} \alpha^{\prime}}{\operatorname{tg} \psi}\left(\frac{\operatorname{tg} \chi+\operatorname{tg} \alpha^{\prime}}{p}+\frac{\operatorname{tg} \varphi}{n} \frac{\partial n}{\partial p}\right) \\
& \frac{\partial \vartheta}{\partial p}=-\frac{\operatorname{tg} \vartheta}{\operatorname{tg} \psi}\left(\frac{\operatorname{tg} \chi+\operatorname{tg} \alpha^{\prime}}{p}+\frac{\operatorname{tg} \varphi}{n} \frac{\partial n}{\partial p}\right)
\end{aligned}
$$

Um endlich noch $\frac{\partial n}{\partial p} \mathrm{zu}$ bestimmen, dient Gleichung 33 , in welcher $G$ constant sein soll, so dass, wenn wir die rechte Seite mit $f(p, n)$ bezeichnen,

$$
\frac{\partial n}{\partial p}=-\frac{\frac{\partial f}{\partial p}}{\frac{\partial f}{\partial n}}=-\frac{n}{p} \frac{\left(n-1+\frac{r}{r^{\prime}}\right)^{2}-\frac{r^{2}}{p^{2} r^{2}}\left(1-\frac{r^{\prime}}{r-\delta}\right)}{p-\left(1-\frac{p-1}{p} \frac{r}{r^{\prime}}\right)\left(1-\frac{p-1}{p} \frac{r}{r^{\prime}}-\frac{r}{p(r-\delta)}\right)}
$$

Setzt man den letzteren Bruch $=N$, so ist

$$
\frac{\partial n}{\partial p}=-\frac{n}{p} N \text {, }
$$

und mit Einsetzung aller Werthe findet man schliesslich

1) Weil $\varepsilon$ constant íst. 


$$
-p \operatorname{tg}^{3} \psi \operatorname{tg}^{2} \chi \frac{\partial C}{\partial p}=
$$

$(\operatorname{tg} \varepsilon-\operatorname{tg} \vartheta)(\operatorname{tg} \psi+\operatorname{tg} \chi)\left[\omega\left(N \operatorname{tg} \varphi+\operatorname{tg} \vartheta-\operatorname{tg} \alpha^{4}\right)-\operatorname{tg} \psi\left(\frac{N \operatorname{tg} \varphi}{\cos ^{2} \varphi}-\frac{\operatorname{tg} \alpha^{2}}{\cos ^{2} \alpha^{4}}\right)\right]$

$$
+\operatorname{tg} \chi(\operatorname{tg} \varepsilon+\operatorname{tg} \psi)\left(N \operatorname{tg} \varphi-\operatorname{tg} \psi-\operatorname{tg} \alpha^{\prime}\right) \frac{\operatorname{tg} \vartheta}{\cos ^{2} \vartheta},
$$

worin zur Abkürzung gesetzt ist

$$
\omega=\frac{\operatorname{tg} \varphi}{\cos ^{2} \varphi}-\frac{\operatorname{tg} \alpha}{\cos ^{2} \alpha}+\frac{\operatorname{tg} \varphi^{\prime}}{\cos ^{2} \varphi^{\prime}}-\frac{\operatorname{tg} \alpha^{\prime}}{\cos ^{2} \alpha^{\prime}}
$$

Aus Gleichung 35 ist das Vorzeichen von $\frac{\partial C}{\partial p}$ nicht leicht zu ïbersehen. Da aber bei continuirlich gesehichteten Linsen an jeder Trennungsfläche $p$ nur unmerklich von 1 verschieden sein kann, so genügt es vollkommen, nur die Frage zu beantworten, welchen Einfluss es auf die Bildgüte hat, wenn $p$ von 1 auf $1 \pm h$ ( $h$ eine unmerklich kleine Grösse) übergeht, d. h. wenn von dem homogenen Zustand der Linse $(p=1)$ ibbergegangen wird auf den geschichteten Zustand, indem an die Fläehe mit dem Radius $r^{\prime}$ ein unmerklich kleines Brechungsverhältniss $1 \pm h$ verlegt wird, und zwar obne Aenderung von $G, r, \delta, \varepsilon$. Um diese Frage zu beantworten, ist einfach in Gleichung 34 und $35 p=1 \mathrm{zu}$ setzen, und das Vorzeichen von $\frac{\partial C}{\partial p}$ zu ermitteln. Es wird dann $\varphi^{\prime}=\alpha^{\prime}$ und $\varepsilon$ und $\vartheta$ nehmen die Werthe an, die sie bei homogener Linse haben würden: $\varepsilon=\vartheta+\varphi-\alpha$ und $(r-\delta) \sin \vartheta=r \sin \alpha$.

Da aber ferner bei continuirlicher Schichtung der Abstand zweier Niveauflächen sehr klein ist, so können wir weiter diesen Abstand als eine unendlich kleine Grösse erster Ordnung setzen, also, wenn $k$ eine solche Grösse bedeutet, annehmen

Nunmehr wird

$$
\frac{r}{r^{\prime}}=1+k \text { oder } \quad r-r^{\prime}=k r^{\prime} \text {. }
$$

worin $R$ zur Abkürzung steht für

$$
N=1+k R
$$

$$
R=\frac{2 n(r-\delta)-(r-2 \delta)}{n^{2}(r-\delta)+\delta}
$$

Ferner wird für die angenommenen Werthe von $p$ und $r^{t}$

$$
\begin{gathered}
\operatorname{tg} \alpha^{\prime}=\operatorname{tg} \varphi^{\prime}=\operatorname{tg} \alpha+k \frac{\operatorname{tg} \alpha}{\cos ^{2} \alpha} \\
\frac{\operatorname{tg} \alpha^{\prime}}{\cos ^{2} \alpha^{\prime}}=\frac{\operatorname{tg} \alpha}{\cos ^{2} \alpha}+k \frac{\operatorname{tg} \alpha}{\cos ^{2} \alpha}-k \frac{\operatorname{tg}^{3} \alpha}{\cos ^{2} \alpha} .
\end{gathered}
$$


Brechung bei schiefer Incidenz, mit besonderer Berücksichtigung d. Auges. 307

Der Werth von $\omega$ beschränkt sich, da $\varphi^{\prime}=\alpha^{\prime}$, auf seine beiden ersten Glieder.

Setzt man diese Werthe in die Gleichung 35 ein, so erhält man durch mehrfache Vereinfachungen (unter Berïcksichtigung von Gl. 24 und 25) die Gleichung

$$
\begin{aligned}
(36) & -\frac{\operatorname{tg}^{3} \psi \operatorname{tg}^{2} \chi}{h} \frac{\partial C}{\partial p}=(\operatorname{tg} \varepsilon-\operatorname{tg} \vartheta)(\operatorname{tg} \psi+\operatorname{tg} \chi)\left\{\left(\frac{\operatorname{tg} \varphi}{\cos ^{2} \varphi}-\frac{\operatorname{tg} \alpha}{\cos ^{2} \alpha}\right)\left(R \operatorname{tg} \varphi-\frac{\operatorname{tg} \alpha}{\cos ^{2} \alpha}\right)\right. \\
& \left.-\operatorname{tg} \psi\left(\frac{R \operatorname{tg} \varphi}{\cos ^{2} \varphi}-\frac{\operatorname{tg} \alpha}{\cos ^{2} \alpha}+\frac{\operatorname{tg}^{3} \alpha}{\cos ^{2} \alpha}\right)\right\}+\operatorname{tg} \chi(\operatorname{tg} \varepsilon+\operatorname{tg} \chi) \frac{\operatorname{tg} \vartheta}{\cos ^{2} \vartheta}\left(R \operatorname{tg} \varphi-\frac{\operatorname{tg} \alpha}{\cos ^{2} \alpha}\right) .
\end{aligned}
$$

Um nun aus dieser völlig strengen Gleichung Schlüsse ziehen zu können, beschränken wir uns auf solche Winkel, dass die 5. Potenz der Tangente gegen die 1. und 3. vernachlässigt werden kann. Entwickelt man den Ausdruck für $\operatorname{tg} \varepsilon\left(\varepsilon=\vartheta+\varphi-\alpha\right.$, da $\varphi^{\prime}=\alpha^{\prime}$; vgl. Gl. 17), so erhält man bis auf Grössen 5. Ordnung genau

$$
\operatorname{tg} \varepsilon=\operatorname{tg} \psi+\operatorname{tg} x(\operatorname{tg} \psi \operatorname{tg} \vartheta-\operatorname{tg} \varphi \operatorname{tg} \alpha)
$$

und weiter folgt hieraus, dass bis auf Grössen 5. Ordnung genan

$$
(\operatorname{tg} \varepsilon-\operatorname{tg} \vartheta)(\operatorname{tg} \psi+\operatorname{tg} x)=\operatorname{tg} \chi(\operatorname{tg} \varepsilon+\operatorname{tg} \chi)
$$

so dass man erhält, indem man beriicksichtigt, dass

$$
\frac{\operatorname{tg} \varphi}{\cos ^{2} \varphi}=\operatorname{tg} \varphi+\operatorname{tg}^{3} \varphi
$$

und dass

$$
\operatorname{tg} \vartheta+\operatorname{tg} \varphi-\operatorname{tg} \alpha=\operatorname{tg} \psi
$$

bis auf Grössen 5. Ordnung genau:

$$
\frac{\operatorname{tg}^{3} \psi \operatorname{tg} \chi}{k(\operatorname{tg} \varepsilon+\operatorname{tg} \chi)} \frac{\partial C}{\partial p}=
$$

$$
\operatorname{tg} \psi\left(R \operatorname{tg}^{3} \varphi+\operatorname{tg}^{3} \alpha\right)-\left(\operatorname{tg}^{3} \vartheta+\operatorname{tg}^{3} \varphi-\operatorname{tg}^{3} \alpha\right)(R \operatorname{tg} \varphi-\operatorname{tg} \alpha) .
$$

Die Untersuchung dieses Ausdrucks ergiebt, dass $\frac{\partial C}{\partial p}$ positiv ist $\left.{ }^{1}\right)$, gewisse extreme Fälle ausgenommen, d. h. dass

eine gegebene Linse bei gegebener Incidenzschiefe $\varepsilon$ ein besseres Bild, als bei homogener Beschaffenheit, dann liefert, wenn man, ohne Veränderung der centralen Brennweite, in die Nähe der äusseren Fläche eine concentrische Grenzfläche mit positivem

1) $R$ ist in allen Fällen ein wenig kleiner als 1 ; die rechte Seite der Gleichung 37 kann erst dann negativ werden, wenn $\delta$ grösser wird als ${ }^{2} / 3 r$, in welchem Falle die Linse schon nahezu Kugelgestalt hätte; dies gilt für alle Werthe von $n$, die unter 2 liegen; für $n=1,2$ müsste sogar $\delta$ grösser als ${ }^{3 / 4} r$ werden, damit die rechte Seite der Gleichung negativ werden kann.

E. PAlüger, Archiv f. Physiologie. Bd, XxvIr. 
Werthe des Brechungsverhältnisses verlegt, während umgekehrf bei negativem Werthe das Bild sich verschlechtert.

Da jede neue Grenzfläche von derselben Art ebenso wirken muss ${ }^{1}$ ), so lässt sich weiter der Satz aufstellen:

Eine Linse von constant bleibender centraler Brennweite und gegebener Incidenzschiefe gewinnt an Bildgüte durch concentrische Schichtung von der Art, dass in concentrischen Niveauflächen das Brechungsvermögen nach innen zunimmt.

Dieser Satz ist in aller Strenge nur innerhalb des allerdings schon recht beträchtlichen Winkelbereiches bewiesen, für welchen $\operatorname{tg}^{5} \varepsilon$ gegen $\operatorname{tg} \varepsilon$ vernachlässigt werden darf. Doch unterliegt es kaum einem Zweifel, dass er auch für noch grössere Winkel gilt, obwohl sich hier der Beweis nicht mehr übersichtlich fübren lässt. Für sehr k le in e Winkel (erster Ordnung) gilt er im Gegentheil noch nicht, wie mir die Untersuchung gezeigt hat; man sieht dies schon aus Gleichung 37, deren rechte Seite $=0$ wird, wenn man die dritte Potenz der Tangenten vernachlässigt; $d . h$. für den ersten Winkelbereich ist die Bildgüte unabhängig von $p$. Das Gleiche ergiebt sich direct aus Gleichung 29, welche, da für Winkel erster Ordnung $\varepsilon=\psi$ wird, übergeht in

$$
\frac{1}{B}=\sin ^{2} \varepsilon \text {. }
$$

Dieselbe Beziehung hatten wir schon früher (Theil II. S. 371) für Linsen von verschwindender $\mathrm{Dick}$, und zwar hier für alle Winkelbereiche gefunden.

Das Resultat lautet also:

Die Bildgite ist unabhängig von der Beschaffenheit der Linse (in Bezug auf Schichtung oder Homogenität), 1. für sehr dünne Linsen, 2. für sehr kleine Incidenzwinkel. Sie wird dagegen in allen gewöhnlichen Fällen durch eine schichtweise Indexzunahme nach Art der Krystallinse vergrössert (bewiesen zunächst für den angegebenen Winkelbereich, für grössere Winkel wahrscheinlich noch gïnstiger).

Die Begiunstigung der Periscopie durch schichtweise Indexzunahme ist im Vorstehenden zunächst für concentrische Schich-

1) Für die innen folgenden Grenzflächen wird $\delta$ im Verhältniss zum Radius immer kleiner, so dass für sie erst recht der Werth $\frac{\partial C}{\partial p}$ positiv sein muss, wenn er es für die erste Fläche war. 
Brechung bei schiefer Incidenz, mit besonderer Berücksichtigung d. Auges. 309

tung bewiesen, für welche sich die Rechnung einfach und sogar elegant gestaltet. Für nicht concentrische Schichtung ist vermuthlich die Begünstigung noch grösser, sobald (wie bei der Krystalllinse) die Krümmungen rascher zunehmen als bei concentrischer Beschaffenheit; denn hierdurch werden die inneren Brechungen immer normaler. Schon friber habe ich darauf hingewiesen, dass eine kuglige Kernbeschaffenheit einen ungemein günstigen Fall darstellt ${ }^{1}$ ), und diesem Verhalten das bezeichnete Krïmmungsverhältniss sich mehr annähert als das concentrische.

\section{Die Differentialgleiohungen für eine Linse mit continuirlicher Indexzunahme.}

Obgleich die von mir tor 8 Jahren aufgeworfene Frage, ob die Schichtung der Krystallinse für die Bilder bei schiefer Incidenz günstig ist, im Vorstehenden bejahend beantwortet ist, und zwar grade für den Fall continuirlicher Indexzunahme, wird es doch nützlich sein, für den letzteren Fall auch directe Gleichungen aufzustellen. Auch hier beschränke ich mich auf concentrische Anordnung der Niveauflächen.

a) Differentialgleichung für den Hauptschnitt.

Der Radius einer beliebigen Niveaufläche sei $\varrho$ (Fig. 4), der Index auf der inneren, concaven Seite $\mu$, auf der äusseren, nach welcher die Radien zunebmen, $\mu+d \mu$; ferner seien die Winkel des durchgehenden Strahles mit dem Einfallsloth wiederum aussen $\varphi$, innen $\alpha$, so dass

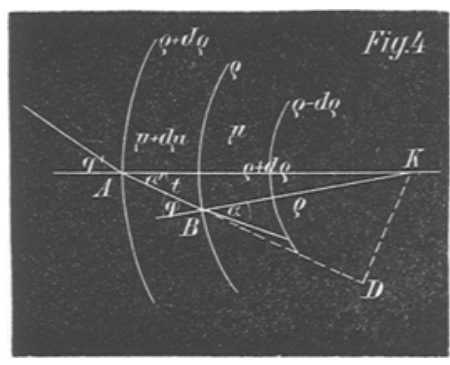

$$
\sin \varphi=\left(1-\frac{d \mu}{\mu}\right) \sin \alpha
$$

1) Bei diesem Anlass muss ich mich gegen einen kleinen Irrthum verwahren; Matthiessen (dies Archiv, Bd. XIX, p. 521) sagt, dass ich die Hypothese aufgestellt habe, der Kern der Krystalllinse sei kuglig. Ich bin aber davon weit entfernt gewesen, sondern habe nur, zu einer Zeit, wo ich noch nicht Gleichungen für dicke geschichtete Linsen aufgestellt hatte, die kuglige Kernform als ein besonders einfaches Beispiel von stärker gekrümmtem Kern der Rechnung unterzogen; es heisst an der betreffenden Stelle (Ueber schiefen Durchgang etc., 1874, p. 20): „Die Formeln werden noch einigermassen übersehbar, wenn man die Kernlinse kugelig annimmt" etc. 
es wird dann, da

$$
\frac{\sin (\varphi-\alpha)}{\sin \alpha}=-\frac{d \mu}{\mu \cos \alpha}
$$

nach Gl. 1:

$$
\frac{1}{e^{\star}}+\left(1-\frac{d \mu}{\mu}\right) \frac{1}{f^{*}}=-\frac{d \mu}{\mu \varrho \cos \alpha} \text {. }
$$

Nun ist aber die vordere Bildweite $e_{*}$ der Fläche $\varrho$ identisch mit der hinteren Bildweite der nächst äusseren Fläche $(\varrho+d \varrho)$, wenn man diese Bildweite negativ nimmt und zugleich um das Stück $A B=t$ vermindert, $\mathrm{d}$. h. es ist

$$
e^{*}=-\left(f^{*}+d f^{*}-t\right) \text {. }
$$

Um den Werth ron $t$ zu finden, beachte man, dass

$$
t=A D-B D=(\varrho+d \varrho) \cos \alpha^{2}-\varrho \cos \varphi,
$$

oder, da $\alpha^{4}=\alpha+d \alpha$ und $\cos \varphi=\cos \alpha+\frac{d \mu}{\mu} \frac{\sin ^{2} \alpha}{\cos \alpha}$,

$$
t=(\varrho+d \varrho)(\cos \alpha-\sin \alpha d \alpha)-\varrho\left(\cos \alpha+\frac{d \mu}{\mu} \frac{\sin ^{2} \alpha}{\cos \alpha}\right)
$$

Zur Bestimmung von $d \alpha$ dient das Dreieck $a b c$, in welchem $\varrho \sin \varphi$ $=(\varrho+d \varrho) \sin \alpha$, also mit Einsetzung der Werthe:

$$
\left(1-\frac{d \mu}{\mu}\right)\left(1-\frac{d \varrho}{\varrho}\right) \sin \alpha=\sin \alpha+\cos \alpha d \alpha
$$

oder

$$
\operatorname{cotg} \alpha d \alpha+\frac{d \mu}{\mu}+\frac{d \varrho}{\varrho}=0 .
$$

Fïhrt man den hieraus folgenden Werth von $d \alpha$ in die Gleichung für $t$ ein, so findet man

$$
t=\frac{d \underline{g}}{\cos \alpha}
$$

so dass nunmehr mit Einsetzung von

$$
e^{\star}=-\left(f^{*}+d f_{\star}-\frac{d \varrho}{\cos \alpha}\right)
$$

in die Gleichung 38 sich ergiebt

$$
\mu \varrho+\varrho \cos \alpha\left(f_{*} \frac{d \mu}{d \varrho}-\mu \frac{d f *}{d \varrho}\right)=f^{2} \frac{d \mu}{d \varrho} .
$$

Ferner ergiebt die Integration der Gleichung 39

$$
\lg \sin \alpha+\lg \mu+\lg \varrho=\text { const., }
$$

$$
\mu \varrho \sin \alpha=k \text {. }
$$


Brechung bei schiefer Incidenz, mit besonderer Berücksichtigung d. Auges. 311

Setzt man dies in Gleichung 40 ein, so ergiebt sich

$$
\mu \varrho+\sqrt{\varrho^{2}-\frac{k^{2}}{\mu^{2}}}\left(f * \frac{d \mu}{d \varrho}-\mu \frac{d f *}{d \varrho}\right)=f * \frac{d \mu}{d \varrho} \text {. }
$$

In dieser Differentialgleichung ist noch die Beziehung zwischen $\mu$ und $\varrho$ disponibel, über welche eine Annahme zu machen ist, etwa den Verhältnissen der Krystalllinse entsprechend.

b) Differentialgleichung für den Kreuzschnitt.

Aus Gleichung 1 ergiebt sich für die Fläche $\varrho$, wenn man die Beziehung zwischen $\varphi$ und $\alpha$ berücksichtigt,

$$
\frac{\cos ^{2} \alpha+2 \frac{d \mu}{\mu} \sin ^{2} \alpha}{e \dagger}+\frac{\left(1-\frac{d \mu}{\mu}\right) \cos ^{2} \alpha}{f \dagger}=-\frac{d \mu}{\mu \rho \cos \alpha},
$$

und da (vgl. oben)

$$
e \dagger=-\left(f \dagger+d f \dagger-\frac{d \varrho}{\cos \alpha}\right)
$$

$$
\mu \varrho \cos ^{2} \alpha+\varrho \cos \alpha\left[f \dagger \frac{d \mu}{d \varrho}\left(1+\sin ^{2} a\right)-\mu \frac{d f \dagger}{d \varrho} \cos ^{2} \alpha\right]=f+\frac{d \mu}{d \varrho}
$$

woraus wiederum der Winkel $\alpha$ mittels der Gleichnng 41 zu eliminiren, und dann die Annahme hinsichtlich der Beziehung zwischen $\mu$ und $\varrho$ einzuführen ist.

c) Differentialgleichung für normalen Durchgang.

Hier wird $\alpha=0$, und die Gleichungen 40 und 44 reduciren sich auf

$$
\mu \varrho+\varrho\left(f \frac{d \mu}{d \varrho}-\mu \frac{d f}{d \grave{\varrho}}\right)=f^{2} \frac{d \mu}{d \varrho}
$$

worin wiederum die Beziehung von $\mu$ und $\varrho$ einzuführen ist. Die Gleichung 45 lässt sich auch leicht direct ableiten.

\section{Integration vorstehender Differentialgleichungen fïr einen Specialfall.}

Die einzuführende willkürliche Beziehnng zwischen $\mu$ und $\varrho$ muss so sein, dass $\mu$ zunimmt, wenn $\varrho$ abnimmt; das betr. Gesetz ist aber für die Krystalllinse nicht näher bekannt. Die einfachste unter allen Annahmen wäre $\mu \varrho=$ const.; sie trifft freilich für die Krystallinse nicht zu, deren Index nicht so rasch zunimmt wie 
die Radien abnehmen, welche übrigens auch nicht concentrisch geschichtet ist; jedoch ist schon die strenge Durchführung eines einzigen Falles lehrreich.

Setzt man also $\mu \varrho=c$, so folgt aus Gl. 41 , dass $\alpha=$ const., d. h. das Strahlenbündel durchläuft alle Niveauflächen unter gleichem Winkel, und bildet eine logarithmische Spirale.

Die Integration gestaltet sich nunmehr folgendermassen, da

$$
\frac{d \mu}{d \varrho}=-\frac{c}{\varrho^{2}} \text { und } \sin \alpha=\frac{k}{c}:
$$

a) für den Hauptschnitt.

Gleichung 40 nimmt die Gestalt an:

oder

$$
c+\varrho \cos \alpha\left(-\frac{c f^{*}}{\varrho^{2}}-\frac{c}{\varrho} \frac{d f^{*}}{d \varrho}\right)=-\frac{c}{\varrho^{2}} f^{2},
$$

$$
1-\cos \alpha\left(\frac{d f^{*}}{d \varrho}+\frac{f^{*}}{\varrho}\right)+\left(\frac{f^{*}}{\varrho}\right)^{2}=0 .
$$

Setzt man, behufs Trennung der Veränderlichen,

$$
\frac{f_{\star}}{\varrho}=z, \quad \text { also } \frac{d f_{\star}}{d \varrho}=\varrho \frac{d z}{d \varrho}+\xi_{2}
$$

so erbält man, nach gehöriger Umformung,

$$
\frac{d \varrho}{\varrho}=\cos \alpha \frac{d z}{1-2 z \cos \alpha+z^{2}},
$$

woraus, da $\cos \alpha$ constant ist, durch Integration folgt

$$
\begin{aligned}
& \lg \varrho=\operatorname{cotg} \alpha \cdot \operatorname{arctg} \frac{z-\cos \alpha}{\sin \alpha}+\text { const. } \\
= & \operatorname{cotg} \alpha \cdot \operatorname{arctg}\left(\frac{f_{*}}{\varrho \sin \alpha}-\operatorname{cotg} \alpha\right)+\text { const. }
\end{aligned}
$$

Zur Bestimmung der Cónstante nennen wir $r$ den Radius der vorderen Grenzfläche und $f^{\prime} *$ den Werth von $f_{*}$ für $\varrho=r$, so dass

also

$$
\lg r=\operatorname{cotg} \alpha . \operatorname{arctg}\left(\frac{f^{*} *}{r \sin \alpha}-\operatorname{cotg} \alpha\right)+\text { const., }
$$

$$
\lg \frac{r}{\varrho}=\operatorname{cotg} \alpha \cdot \operatorname{arctg} \frac{\left(\frac{f_{*}^{*}}{r}-\frac{f^{*}}{\varrho}\right) \sin \alpha}{1+\left(\frac{f_{*}^{*}}{r}-\frac{f_{*}}{\varrho}\right) \cos \alpha+\frac{f^{*} * \frac{f_{*}}{r}}{\varrho}} ;
$$

oder wenn man setzt

$$
\lg \frac{r}{\varrho} \cdot \operatorname{tg} \alpha=\lambda
$$


Brechung bei schiefer Incidenz, mit besonderer Berücksichtigung d. Auges. 313

$$
\frac{\left(\frac{f^{*}}{r}-\frac{f^{*}}{\varrho}\right) \sin \alpha}{1+\left(\frac{f^{*}}{r}-\frac{f^{*}}{\varrho}\right) \cos \alpha+\frac{f^{*}}{r} \frac{f_{*}}{\varrho}}=\operatorname{tg} \lambda
$$

Die geometrische Bedeutung von $\lambda$ ergiebt sich aus den Eigenschaften der logarithmischen Spirale, welche in unserem Falle vom Strable gebildet wird. Die Polargleichung einer solchen Spirale ist nämlich, wenn $\omega$ der Polarwinkel, $\varrho$ der Radius Vector, $\alpha$ der constante Winkel zwischen Vector and Tangente, endlich $r$ der. Werth von $\varrho$ für $\omega=0$,

$$
\varrho=r e^{-\omega \operatorname{cotg} \alpha},
$$

woraus folgt

$$
\omega=\lg \frac{r}{\varrho} \cdot \operatorname{tg} \alpha .
$$

Ist also in Figur $5 L A$ das einfallende Bündel, $A D$ (Radius $r$ ) und $A^{\prime} D^{\prime}$ (Radius $\varrho$ ) die Grenzen des geschichteten Systems und $A B$ das spiralige Curvenstück, welches das Bündel im System zurücklegt, so entspricht der Winkel $A K B$ der Bedentung von $\omega$, und sein Werth ist, wie

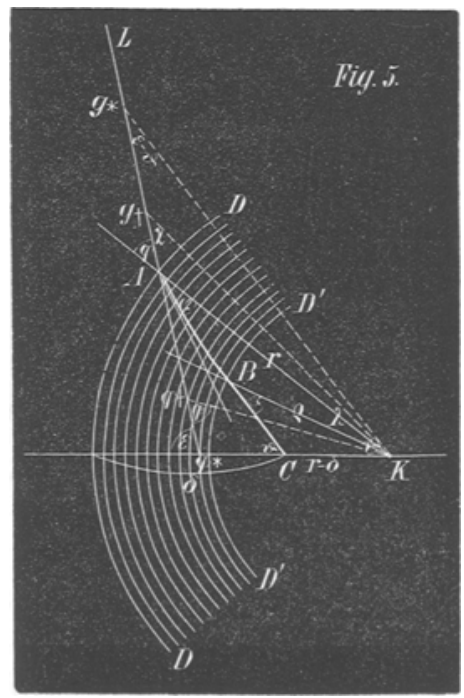
$\operatorname{man}$ sieht, $=\lambda$.

b) für den Kreuzschnitt.

Gleichung 44 geht für $\mu \varrho=e$ über in

$$
\cos ^{2} \alpha-\cos \alpha\left(\frac{f \dagger}{\varrho}\left(1+\sin ^{2} \alpha\right)+\frac{d f \dagger}{d \varrho} \cos ^{2} \alpha\right)=-\frac{f \dagger^{2}}{\varrho^{2}} .
$$

Setzt man behufs Integration

$$
\frac{f \dagger}{\varrho \cos \alpha}=z, \text { also } \frac{d f \dagger}{d \varrho}=\cos \alpha\left(\varrho \frac{d z}{d \varrho}+z\right),
$$

so ergiebt sich

$$
\begin{aligned}
& (1-z)^{2}=\varrho \cos ^{2} \alpha \frac{d z}{d \varrho} \\
& \text { oder } \quad \frac{d \varrho}{\varrho}=\cos ^{2} \alpha \frac{d z}{(1-z)^{2}} .
\end{aligned}
$$


Die Integration ergiebt

$$
\lg \varrho=\frac{\varrho \cos ^{2} \alpha}{1-z}+\text { const. }=\frac{\varrho \cos ^{3} \alpha}{\varrho \cos \alpha-f \dagger}+\text { const. }
$$

Setzt man wiederum für $\varrho=r, f_{\dagger} \dagger=f^{\prime} \dagger$, so folgt

$$
\lg r=\frac{r \cos ^{3} \alpha}{r \cos \alpha-f^{\prime} \dagger}+\text { const., }
$$

also

$$
\lg \frac{r}{\varrho}=\cos ^{3} \alpha \cdot \frac{\frac{f^{\prime} \dagger}{r}-\frac{f \dagger}{\varrho}}{\left(\frac{f^{\prime} \dagger}{r}-\cos \alpha\right)\left(\frac{f^{\dagger}}{\varrho}-\cos \alpha\right)}
$$

oder mit Beriicksichtigung von G1. 47

$$
\frac{\frac{f^{\prime} \dagger}{r}-\frac{f \dagger}{\varrho}}{\left(\frac{f^{\prime} \dagger}{r}-\cos \alpha\right)\left(\frac{f \dagger}{\varrho}-\cos \alpha\right)}=\frac{\lambda}{\sin \alpha \cos ^{2} \alpha} .
$$

c) für den normalen Durehgang.

Setzt man $\alpha=0$, so folgt aus Gl. 46 oder 49 (oder auch ableitbar aus Gl. 45, indem man $\mu \varrho=c$ setzt):

$$
\frac{\frac{f}{r}-\frac{f}{\varrho}}{\left(\frac{f}{r}-1\right)\left(\frac{f}{\varrho}-1\right)}=\lg \frac{r}{\varrho} \text {. }
$$

16. Berechnung der Brennweiten und der Bildgüte für eine Linse von der angenommenen continuirlichen Schichtung.

Wir nehmen wieder die Existenz eines optischen Mittelpunktes (vergl. oben S. 290) und Durchgang der Strahlen durch denselben an. Die Schichtung erstrecke sich vom Radius $r$ bis zum Radius $\varrho$, innerhalb des letzteren bleibe der zugehörige Index bis zum optischen Mittelpunkt, so dass $\varrho$ die Grenze eines homogenen Kerns bedeutet. Jenseits $r$ sei der Index 1, und die Schichtung beginne bei $r$ mit dem Index $n$, so dass also der Kern den Index hat $n \frac{r}{\varrho}$. Endlich sei wieder $\delta$ der Abstand des optischen Mittelpunktes von der ersten Linsenfläche.

Nennt man $e^{*}$, e†, $e$ die Abstände des leuchtenden Punktes 
Brechung bei schiefer Incidenz, mit besonderer Berücksichtigung d. Auges. 315

von der 1. Linsenfläche, so findet vermöge der Brechung an dieser Fläche die durch die Gleichungen 1 gegebene Beziehung statt zwischen $e^{*}$ and $f^{\prime} *$ (Gl. 48), e† und $f^{\prime} \dagger$ (Gl. 50) etc., und man kann so direct Beziehungen finden (unter Elimination von $f^{*}{ }^{*}, f^{\dagger} \dagger$ ) zwisehen den conjugirten Bildweiten, die vordere gemessen yon der 1. Linsenfläche $(r)$ nach vorn, die hintere von der Kernfläche (e) nach hinten. Man findet so

aus 1 und 48 , in $1 f^{*}$ für $f^{*}$ eingesetzt:

$$
\frac{\sin \varphi}{\sin (\varphi-\alpha)-\frac{r}{e^{\star}} \sin \alpha}=\frac{\frac{\varrho}{f_{*}} \sin \lambda+\sin (\alpha-\lambda)}{\frac{\varrho}{f^{*}} \sin (\alpha+\lambda)-\sin \lambda} ;
$$

aus 1 und 50, in $1 f^{\prime} \dagger$ für $f \dagger$ eingesetzt:

$$
\frac{\sin \varphi \cos \alpha}{\sin (\varphi-\alpha)-\frac{r}{e \dagger} \cos ^{2} \varphi \sin \alpha}=\frac{\lambda\left(1-\frac{\varrho}{f \dagger} \cos \alpha\right)-\sin \alpha \cos \alpha}{\lambda\left(1-\frac{\varrho}{f \dagger} \cos \alpha\right)-\frac{\varrho}{f \dagger} \cos ^{2} \alpha \sin \alpha}
$$

endlich aus diesen, mit $\alpha=0$, oder aus 51 :

$$
\frac{n}{n-1-\frac{r}{e}}=\frac{\lg \frac{r}{\varrho}\left(1-\frac{\varrho}{f}\right)-1}{\lg \frac{r}{\varrho}\left(1-\frac{\varrho}{f}\right)-\frac{\varrho}{f}}
$$

Setzt man in diesen drei Gleichungen $f=\infty$ und löst für $e$, so erhält man die Abstände der vorderen Brennpunkte des vorderen Systems $\left(g^{*}, g^{\dagger}, g\right)$; setzt man in denselben Gleichungen für $f$ ein

$$
C B=e^{\frac{\sin (\vartheta-\alpha)}{\sin \alpha}}=\varrho \cos \alpha-(r-\delta) \cos \vartheta
$$

und löst für $e$, so erhält man die Abstände der vorderen Hauptpunkte von der 1 . Fläche $\left(\varphi^{\star}, \varphi_{\dagger} \dagger, \varphi\right)$, und hat dann nur die Werthe $G^{*}=g_{*}-\varphi_{*}$ etc. zu bilden.

Führt man diese Rechnung aus, was hier nicht in extenso dargestellt wird, so gelangt man nach vielfachen vereinfachenden Umformungen zu den Ausdricken

$$
G^{*}=\frac{r \sin \varphi}{\sin (\varphi-\alpha+\lambda)[\cos (\varphi-\alpha+\lambda)+\operatorname{cotg} \vartheta \sin (\varphi-\alpha+\lambda)]}
$$

$$
=\frac{r \sin \vartheta \sin \varphi \cos ^{2} \varphi \cos ^{4} \alpha}{[\cos \alpha \sin (\varphi-\alpha)+\lambda \cos \varphi][\cos \alpha(\cos \vartheta \sin (\varphi-\alpha)+\sin \vartheta \cos \varphi \cos \alpha)+\lambda \cos \vartheta \cos \varphi]}
$$


(57)

$$
G=\frac{n r \varrho}{\varrho\left(n+\lg \frac{r}{\varrho}\right)\left(n-1+\lg \frac{r}{\varrho}\right)+(r-\varrho-d)\left(n-1+\lg \frac{r}{\varrho}\right)^{2}} .
$$

Die Gleichungen 55 und 56 vereinfachen sich aber noch sehr bedeutend, wenn man den Incidenzwinkel $\varepsilon$ einfuhrt. Aus der Figur 5 ersieht man leicht, dass

$$
\varepsilon=9+\varphi-\alpha+\lambda \text {, }
$$

wodurch man erhält $($ da $\varphi-\alpha+\lambda=\varepsilon-9)$

$$
G_{*}=r \cdot \frac{\sin \varphi}{\sin \varepsilon \cos \varepsilon} \cdot \frac{\operatorname{tg} \vartheta}{\operatorname{tg} \varepsilon-\operatorname{tg} \vartheta}
$$

(59) $G \uparrow=r \sin \varphi \cdot \frac{\operatorname{tg} \vartheta}{\left(\operatorname{tg} \varphi-\operatorname{tg} \alpha+\lg \frac{r}{\varrho} \cdot-\frac{\operatorname{tg} \alpha}{\cos ^{2} \alpha}\right)\left(\operatorname{tg} \vartheta+\operatorname{tg} \varphi-\operatorname{tg} \alpha+\lg \frac{r}{\varrho} \cdot \frac{\operatorname{tg} \alpha}{\cos ^{2} \alpha}\right)}$.

Gleichung 58 ist identisch mit Gleichung 26, und Gleichung 59 wird identisch mit Gleichung 27, wenn man setzt

(60) $\operatorname{tg} \varphi-\operatorname{tg} \alpha+\lg \frac{r}{\varrho} \frac{\operatorname{tg} \alpha}{\cos ^{2} \alpha}=\operatorname{tg} \chi$, und $\operatorname{tg} \vartheta+\operatorname{tg} \psi=\operatorname{tg} \psi$.

In der That ergiebt sich, dass das Glied $\lg \frac{r}{\rho} \frac{\operatorname{tg} \alpha}{\cos ^{2} \alpha}$ nichts anderes ist, als die Summe oder das Integral aller Differenzen zwischen den Tangenten des Einfalls- und Brechungswinkels an jeder Niveaufläche, während $\operatorname{tg} \varphi$ - $\operatorname{tg} \alpha$ diese Differenz für die 1. Linsenfläche darstellt. Da der Brechungswinkel überall $=\alpha$ ist, so ist der Einfallswinkel $\xi$ an einer Niveaufläche bestimmt durch

$$
\sin \xi=\left(1-\frac{d \mu}{\mu}\right) \sin \alpha=\left(1+\frac{d \varrho}{\varrho}\right) \sin \alpha
$$

woraus folgt

$$
\operatorname{tg} \xi=\operatorname{tg} \alpha\left(1+\frac{d \varrho}{\varrho \cos ^{2} \alpha}\right) \text { oder } \operatorname{tg} \xi-\operatorname{tg} \alpha=\frac{d \varrho}{\varrho} \frac{\operatorname{tg} \alpha}{\cos ^{2} \alpha} .
$$

Integrirt man diesen Werth von $\varrho$ bis $r$, so ergiebt sich, wie nach Gl. 30 zu erwarten war,

$$
\int_{\varrho}^{r}(\operatorname{tg} \xi-\operatorname{tg} \alpha) d \varrho=\lg \frac{r}{\varrho} \cdot \frac{\operatorname{tg} \alpha}{\cos ^{2} \alpha} .
$$

Als Ausdruck für die Bildgï te gilt also auch hier die Gleichung 29, wenn man für $\psi$ und $\chi$ die durch Gleichung 60 bestimmten Werthe einsetzt.

Nach dem S. 308 Gesagten muss die Bildgüte einer solchen Linse grösser sein, als die einer homogenen Linse von gleicher 
centraler Brennweite (diese letztere durch Gl. 57 bestimmt); dies bestätigt sich durch numerische Beispiele, und zwar auch für die allergrössten Incidenzwinkel.

\section{Die Gleichungen für eine Linse mit continuirlicher Indexänderung belieblger Art in concentrischen Niveaufiächen.}

In den beiden vorigen Paragraphen sind die Differentialgleichungen der continuirlich geschichteten Linse nur für eine ganz specielle Annahme bezüglich des Verhältnisses zwischen $\mu$ und $\varrho$ integrirt worden (vgl. S. 311 f.). Wir haben aber nummehr ein Mittel, die strengen Gleichungen für Brennweiten und Bildgüte direct für jedes Schichtungsgesetz aufzustellen, indem wir die Gleichung 30 benutzen. In dieser hängt $\operatorname{tg} \varphi-\operatorname{tg} \alpha$ vom Brechungsverhältniss an der vorderen Linsenfläche ab, dagegen stellen die folgenden Glieder ein Integral dar, welches die Differenzen der Tangenten des Einfalls- und Brechungswinkels an allen Niveauflächen bis zur Grenze des Kerns umfasst. Um diesen Integralwerth aufzustellen, beachte man, dass an jeder Niveaufläche (vgl. S. 309)

$$
\sin \varphi^{\prime}=\left(1-\frac{d \mu}{\mu}\right) \sin \alpha^{\prime},
$$

woraus sich ableitet

also

$$
\operatorname{tg} \varphi^{\prime}=\operatorname{tg} \alpha^{\prime}\left(1-\frac{d \mu}{\mu \cos ^{2} \alpha^{\prime}}\right)
$$

$$
\operatorname{tg} \varphi^{\prime}-\operatorname{tg} \alpha^{\prime}=-\frac{d \mu}{\mu} \frac{\operatorname{tg} \alpha^{\prime}}{\cos ^{2} \alpha^{\prime}},
$$

wofür man, unter Berïcksichtigung von Gleichung 39 , anch setzen kann

so dass (Gleichung 30)

$$
\operatorname{tg} \varphi^{\prime}-\operatorname{tg} \alpha^{\prime}=\frac{\operatorname{tg} \alpha^{\prime}}{\cos ^{2} \alpha^{\prime}} \frac{d \varrho}{\varrho}+\frac{d \alpha^{\prime}}{\cos ^{2} \alpha^{\prime \prime}}
$$

$$
\operatorname{tg} \chi=\operatorname{tg} \varphi-\operatorname{tg} \alpha+\int_{\varrho}^{r}\left(\frac{\operatorname{tg} \alpha^{4}}{\cos ^{2} \alpha^{\prime}} \frac{d \varrho}{\varrho}+\frac{d \alpha^{\prime}}{\cos ^{2} \alpha^{\prime}}\right) .
$$

Unter dem Integralzeichen lässt sich mittels Gleichung 41, wonach

$$
\mu \varrho \sin \alpha^{\prime}=k,
$$

sowohl $\alpha^{\prime}$ als $d \alpha^{\prime}$ auf Functionen von $\varrho$ reduciren, sobald eine bestimmte Annahme über die Beziehung zwischen $\mu$ und $\varrho, d$. h. das Gesetz der Indexänderung, gemacht ist. 
Ferner ist stets auch 9 und $\varepsilon$ leicht zu bestimmen; es muss nämlich immer sein (vgl. Fig. 3 oder 5)

$$
\varrho \sin \alpha^{\prime \prime}=(r-\delta) \sin \vartheta
$$

wenn man $\varrho$ den Radius der Kernfläche und $\alpha^{\prime \prime}$ den Werth des Brechungswinkels für diese Fläche nennt, der sich stets aus dem Vorstehenden ermitteln lässt. Ebenso ist stets, wie man aus Fig. 5 entnehmen kann,

$$
\varepsilon=\vartheta+\varphi-\alpha^{\prime \prime}+\lambda,
$$

wenn $\alpha^{\prime \prime}$ die eben angegebene Bedeutung hat ${ }^{1}$, und $\lambda$ den Winkel zwischen dem ersten und letzten Einfallsradius bezeichnet; auch dieser Winkel ist immer angebbar. Da nunmehr alle Daten für die Aufstellung der Gleichungen 30,31 und 26-29 vorhanden sind, so lassen für alle Schichtungsannahmen, sobald dieselben integrirbare Ausdrücke ergeben, Brennweiten und Bildgüte sich genau berechnen. Die centrale Brennweite ist ebenfalls dann immer leieht zu finden, indem man die Winkel verschwinden lässt.

\section{Schlussbemerkungen.}

Der Weg, noch andere Arten geschichteter Linsen auf die Bildguite bei schiefem Durchgang zu untersuchen, ist in dieser Arbeit so vollständig vorgezeichnet, dass Jeder, der die Aufgabe weiter verfolgen will, die Anknüpfungspunkte vorfindet. Der nächste Schritt wäre der Uebergang zur nicht concentrischen Schichtung; ist in diesem Falle der Abstand der Krümmungsmittelpunkte nach Grösse und Vorzeichen gegeben, so lassen sich ohne Schwierigkeit Gleichungen wie No. 13-17 aufstellen, welche aber, ebenso wie die aus ihnen abgeleiteten Ausdrücke für $G *, G \dagger$ etc., ungemein complicirt ausfallen.

Es erscheint unnöthig, diese Berechnungen hier auszuführen, da die prineipielle Frage bereits entschieden ist. Schon bei concentrischer Schichtung ist das Bild für den Schichtungsfall innerhalb weiter Grenzen besser als für die gleichwerthige homogene Linse, sobald, wie bei der Krystalllinse, der Brechungsindex nach innen zunimmt; für nichtconcentrische Schichtung muss dies, wie schon angefithrt, noch allgemeiner der Fall sein, sobald wie bei der Krystallinse die Krümmung rascher zunimmt als im concentrischen System.

1) Bei dem in dem vorigen Paragraphen behandelten Falle war $\alpha$ constant, und daher $\alpha^{\prime \prime}=\alpha$. 
Als ich im Jahre 1874 darauf hinwies, dass die Schichtung der Krystalllinse das Auge periscopischer mache, konnte ich nur für unendlich dünne Linsen die schiefwinklige Abbildung berechnen. Es ergab sich, dass ganz allgemein solche Linsen homocentrischere Bilder, d. h. kürzere Brennstrecken geben, als gleichwerthige homogene Linsen ${ }^{1}$ ). Allein später fand ich, dass die Bildgïte dadurch noch nicht verbessert wird, weil in gleichem Masse wie die Brennstrecke auch die Grösse des Bildes durch die Schichtung verkleinert wird. Der richtig bemessene Werth der Bildgüte zeigt sich für unendlich dünne Linsen unabhängig von der Schichtung ${ }^{2}$ ). Seitdem ist es mir gelungen, auch dicke geschichtete Linsen in voller Strenge zu berechnen und nun zeigt sich, dass diese, wenn sie nach Art der Krystalllinse geschichtet sind, in der That grössere Bildgüte, also grössere Periscopie haben als gleichwerthige homogene Linsen. Dieser Vorzug wird erst für unendlich dünne Linsen, oder für unendlich kleine Incidenzschiefe gleich Null (vgl. S. 308).

Unterdessen ist in meinem Laboratorium von M. P e s c h el der Grad der Periscopie des menschlichen Auges, sowie der isolirten Krystallinse direct empirisch bestimmt, und in der That wesentlich grösser als der gleichwerthiger homogener Systeme gefunden worden ${ }^{3}$ ). Die vorstehenden Berechnungen liefern zum Verständniss dieser Thatsache den Schlüssel. Freilich ist ron Matthiessen und dessen Schïlern noch auf ein andres Moment hingewiesen worden, welches die Periscopie des Auges begünstigt, nämlich auf die Abweichung der brechenden Flächen von der Kugelgestalt ${ }^{4}$ ). Ich bin weit entfernt, die Mitwirkung dieses Momentes zu bezweifeln, zumal wir ja auch auf anderen Gebieten zahlreiche Organisationseigenthümlichkeiten zu demselben Resultate zusammenwirken sehen; indess scheint mir das von mir hervorgehobene Moment das entschieden wirksamere und in der Thierwelt am allgemeinsten verwirklichte.

1) "Ueber schiefen Durchgang etc." Gratulationsschrift für Ludwig. Zürich 1874, p. $18 \mathrm{ff}$; ferner Poggendorff's Annalen, Bd. CLIII, p. 470.

2) Dies Archiv, Bd. XX, p. 371.

3) Dies Archiv, Bd. XVII, p. 504 und Bd. XX, p. 338.

4) Dies Archiv, Bd. XIX, p. 522 und Bd. XX, p. 264. 